\title{
474757 - LOCAL ANESTHETIC DILUTION FOR PERIPHERAL NERVE BLOCK: COMPARATIVE EVALUATION OF SALINE VS 5\% DEXTROSE
}

\author{
Shalini Dhir, MD, Sugantha Ganapathy, FRCPC, Amna Masood, MD \\ Anesthesia and Perioperative Medicine, University of Western Ontario, London, \\ ON, Canada
}

Introduction: Sodium concentration is higher in the saline (NS) diluted drugs. Hypernatrosis is known to antagonize the analgesic effect of local anesthetic (LA) due to sodium channel blockade(1). 5\% dextrose (D5W) is painless on injection and does not cause any long-term sequel in animals or humans when injected around neurological tissue (2). The objective is to prospectively compare and evaluate the onset and duration of action of LA drug when diluted with D5W or NS in patients scheduled for upper limb surgery under peripheral nerve (axillary) block. The results of the pilot study have been analyzed and are being presented.

Methods: After IRB approval \& patient consent, 40 patients scheduled for upper limb surgery under axillary brachial plexus block were enrolled in a double blind pilot study and randomized into two treatment groups. All blocks were done under ultrasound guidance with $0.5 \%$ ropivacaine. Group 1 received LA diluted with D5W and group 2 received LA diluted with NS. Motor and sensory block was tested every 5 minutes for the next 30 minutes. Postoperatively, telephonic interview was conducted to document side effects, satisfaction and time of block resolution.

Results: There were no differences between the groups with respect to demographics. The difference in the time of onset of motor block in both groups was not significant. However, the mean time for onset of complete sensory block was 22 min in saline group while it was 15 min in D5W group and this was significant ( $p$ value $<0.01$ ). The time taken for block recession was 769 minutes in D5W group and 652 minutes in NS group but not significant.

Discussion: Influence of dilution agent on the outcome of peripheral regional block is currently unknown. These pilot data suggest results that D5W dilution is a safe and effective method of dilution of LA and reduces the time of onset of regional block. References: 1 . Kanai A, Hoka S. A comparison of epidural blockade produced by plain $1 \%$ lidocaine and $1 \%$ lidocaine prepared by dilution of $2 \%$ lidocaine with the same volume of saline. Anesth Analg 2006; 102: 1851-5. 2. Tsui BCH, Kropelin B. The electrophysiological effects of dextrose $5 \%$ in water on single shot peripheral nerve stimulation. Anesth Analg 2005; 100: 1837-39. 
Interaction Bar Plot for complete sensory block

Efect: Group

Error Bars : $95 \%$ Con fiden ce Interval

Row exclusion: D5Wstudy.sud

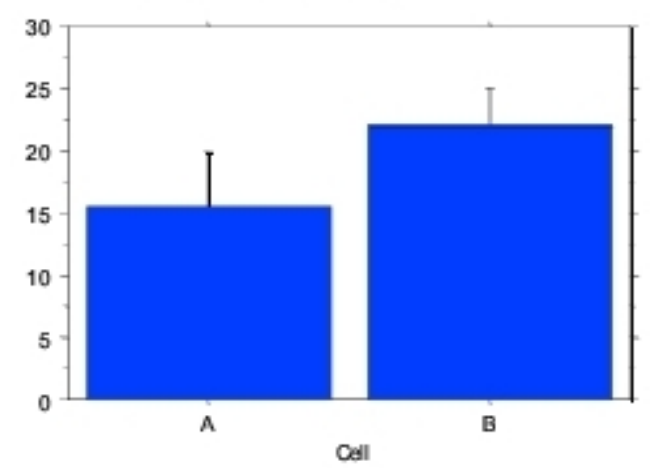

